\title{
Attitudes of Australian health professionals towards rapid genomic testing in neonatal and paediatric intensive care
}

\author{
Zornitza Stark ${ }^{1,2,3} \cdot$ Amy Nisselle ${ }^{1,2,3} \cdot$ Belinda McClaren $\mathbb{1}^{1,2,3} \cdot$ Fiona Lynch $\mathbb{1}^{1,2,3} \cdot$ Stephanie Best ${ }^{4}$. \\ Janet C. Long $\mathbb{1}^{4} \cdot$ Melissa Martyn ${ }^{2,3,5}$. Chirag Patel ${ }^{6} \cdot$ Luregn J. Schlapbach $^{7,8,9,10}$ - Christopher Barnett ${ }^{11}$. \\ Christiane Theda ${ }^{2,3,12} \cdot$ Jason Pinner ${ }^{13} \cdot$ Marcel E. Dinger $\mathbb{I}^{14,15,16} \cdot$ Sebastian Lunke $\mathbb{I D}^{1,2,3} \cdot$ Clara L. Gaff $^{1,2,5,17}$
}

Received: 2 January 2019 / Revised: 10 April 2019 / Accepted: 30 April 2019 / Published online: 31 May 2019

(c) European Society of Human Genetics 2019

\begin{abstract}
We investigated the attitudes of intensive care physicians and genetics professionals towards rapid genomic testing in neonatal and paediatric intensive care units (NICU/PICU). A mixed-methods study (surveys and interviews) was conducted at 13 Australian hospitals and three laboratories involved in multi-center implementation of rapid genomic testing. We investigated experience and confidence with genomic tests among intensivists; perceived usefulness of genomic diagnostic results; preferences for service delivery models; and implementation readiness among genetic services. The overall survey response rate was 59\%, 47\% for intensivists (80/170), and 75\% (91/121) for genetics professionals. Intensivists reported moderate confidence with microarray tests and lower confidence with genomic tests. The majority of intensivists (77\%), clinical geneticists $(87 \%)$ and genetic counsellors $(82 \%)$ favoured a clinical genetics-led service delivery model of genomic testing. Perceived clinical utility of genomic results was lower in the intensivist group compared to the genetics professionals group (20 v 50\%, $p<0.001)$. Interviews ( $n=6$ intensivists; $n=11$ genetic counselors) demonstrated support for implementation, with concerns relating to implementation environment and organizational readiness. Overall, our findings support initial implementation of genomic testing in NICU/PICU as part of an interdisciplinary service delivery model that promotes gradual adoption of genomics by the intensive care workforce while ensuring safety, sustainability, and efficiency.
\end{abstract}

Supplementary information The online version of this article (https:// doi.org/10.1038/s41431-019-0429-y) contains supplementary material, which is available to authorized users.

Zornitza Stark

zornitza.stark@vcgs.org.au

Australian Genomics Health Alliance, Melbourne, Australia

2 University of Melbourne, Melbourne, Australia

3 Murdoch Children's Research Institute, Melbourne, Australia

4 Australian Institute of Health Innovation, Macquarie University, Sydney, Australia

5 Melbourne Genomics Health Alliance, Melbourne, Australia

6 Genetic Health Queensland, Royal Brisbane and Women's Hospital, Brisbane, Australia

7 Paediatric Critical Care Research Group, Child Health Research Centre, The University of Queensland, Brisbane, Australia

8 Paediatric Intensive Care Unit, Queensland Children's Hospital, Brisbane, Australia

\section{Introduction}

Rapid genomic diagnosis is key to delivering the benefits of precision medicine in neonatal and paediatric intensive care units (NICU/PICU) [1]. Data from multiple small cohorts of

9 Faculty of Medicine, The University of Queensland, Brisbane, Australia

10 Department of Pediatrics, Bern University Hospital, Inselspital, University of Bern, Bern, Switzerland

11 Paediatric and Reproductive Genetics Unit, Women's and Children's Hospital, North Adelaide, Australia

12 Neonatal Services, Royal Women's Hospital, Melbourne, Australia

13 Sydney Children's Hospital, Sydney, Australia

14 Garvan Institute of Medical Research, Sydney, Australia

15 Genome.One, Sydney, Australia

16 St Vincent's Clinical School, University of New South Wales Sydney, Sydney, Australia

17 Walter and Eliza Hall Institute, Melbourne, Australia 
NICU/PICU patients with rare genetic conditions have demonstrated high diagnostic and clinical utility of genomic testing in this setting, and cost-effectiveness [2-10]. Rapid turnaround times of 2-21 days are becoming routinely achievable, and are reported to improve clinical utility of results $[3,5,7-10]$.

Most rapid genomic testing studies to date have been conducted at single tertiary paediatric centers with on-site genomic laboratories, and are not typical of most NICU/ PICU services. Widespread implementation of genomic testing in NICUs and PICUs requires development of clinical and laboratory pathways to deliver genomic testing reliably, cost-efficiently and in a timely manner across multi-centre networks. Furthermore, a broad range of medical specialists, including intensive care physicians [11], now have access to genomic testing. Given the specialized nature of genomic testing and result interpretation, increased costs associated with rapid testing, and potential for results to influence time-critical treatment decisions, there is a need for careful planning to ensure the intensive care workforce is adequately prepared and supported during widespread implementation.

Attitudes of intensive care physicians and genetic professionals towards applying rapid genomic testing (exome sequencing (ES) or genome sequencing (GS)) in NICUs/ PICUs have not previously been investigated, and the readiness of clinical and laboratory genetic services to implement such programs is unknown. A qualitative study involving clinicians caring for critically ill children with congenital heart disease reported moral distress in providers who have to make high-stakes, irrevocable decisions involving genomic results, which they only partially understood [11].

Before commencing multi-center implementation of rapid genomic testing, we used established frameworks to determine views of participating intensive care physicians and genetic professionals regarding usefulness of genomic results and preferences for service delivery models. We sought to ascertain readiness for implementation among genetic professionals, and explored experiences and confidence of intensive care physicians with genomic testing.

\section{Materials and methods}

A mixed-methods, explanatory, sequential design was employed, comprising an initial survey with follow-up interviews [12].

\section{Participants}

Participating sites included 13 hospitals (12 NICUs and six PICUs), all with clinical genetics services on-site; and three laboratories. All intensive care physicians/advanced trainees $(n=170)$, and genetic professionals $(n=121)$ were invited to complete an anonymous online survey in March-April 2018. The genetic professionals group included clinical geneticists/advanced trainees $(\underline{n}=57)$, genetic counselors $(\mathrm{n}=33)$, and laboratory genetic scientists $(\mathrm{n}=31)$. The survey had a plain language statement and completion implied informed consent; participants could provide contact details for follow-up interviews.

\section{Survey design}

Questions were developed through review of literature and published tools [13-16]. The survey included Likert-based questions on intensivist confidence in initiating chromosomal microarray (CMA) and genomic testing, interpreting results, and discussing testing and results with families. All groups were asked about perceived clinical utility of genomic results in NICU/PICU. Clinicians (intensive care physicians, clinical geneticists, and genetic counselors) were asked about their preferred model for service delivery of genomic testing in NICU/PICU. Implementation readiness among clinical and laboratory genetics services was measured using an adapted IGNITE network pre-implementation provider questionnaire (https://ignite-genomics.org/spark/provider-surveys/) and the validated Organizational Readiness for Change questionnaire (ORIC), respectively [17]. Respondents could provide additional comments about their preferred model of practice or general comments. The survey was administered via REDCap online software, with an estimated time to completion of $<10$ min (summarized in Table 1, and available as Supplementary material).

\section{Survey analysis}

Intensivist confidence: median and interquartile range of confidence with CMA and ES/GS clinical task variables were calculated due to non-normal distribution. Utility analysis: intensivist responses were compared to those of clinical geneticists. Perceived utility was determined by respondents indicating in what proportion of NICU/PICU patients tested results from rapid genomic testing would contribute to patient management. Response categories covered deciles or 'Don't know'. Distribution of the utility scores was non-parametric. Median utility scores were calculated and quantile regression performed to determine the confidence interval and p-value around the observed difference in median scores. Differences were investigated using Wilcoxon-sign-rank test for paired confidence observations (confidence in same clinical tasks for each test type), or Chi-square tests for categorical variables. P-values are reported for results where $p<0.05$. Analyses were performed using Stata 15.1 (Stata, College Station, TX, 
Table 1 Summary of survey instruments administered to the different professional groups

\begin{tabular}{|c|c|c|c|c|}
\hline & Intensivists & $\begin{array}{l}\text { Clinical } \\
\text { geneticists }\end{array}$ & $\begin{array}{l}\text { Genetic } \\
\text { counselors }\end{array}$ & $\begin{array}{l}\text { Laboratory genetic } \\
\text { scientists }\end{array}$ \\
\hline Demographics & Yes & Yes & Yes & Yes \\
\hline Perceived clinical utility & Yes & Yes & Yes & Yes \\
\hline Clinical service delivery model & Yes & Yes & Yes & \\
\hline $\begin{array}{l}\text { Experience and confidence with } \\
\text { CMA and ES/GS }\end{array}$ & Yes & & & \\
\hline \multicolumn{5}{|l|}{ Implementation readiness } \\
\hline $\begin{array}{l}\text { - Pre-implementation provider } \\
\text { questionnaire (IGNITE network) }\end{array}$ & & Yes & Yes & \\
\hline $\begin{array}{l}\text { - Organizational readiness } \\
\text { for change }\end{array}$ & & & & Yes \\
\hline
\end{tabular}

USA). Optional free-text responses were reviewed for content by BM, FL, ZS and are illustratively reported to explain quantitative results.

\section{Explanatory interviews}

As the professional groups likely to experience significant clinical practice changes with the implementation of rapid genomic testing, intensivists and genetic counselors were invited for semi-structured interviews. These were audiorecorded, transcribed verbatim, and analysis managed using NVivo 11 software (QSR International Pty Ltd, version 11, 2012). A coding framework was based on broad survey domains initially and further codes added in an inductive process by BM and FL [18].

\section{Results}

\section{Response rate}

Of 291 professionals who received the survey, 80 (47\%) intensivists, 47 clinical geneticists (82\%), 23 (70\%) genetic counsellors, and $21(68 \%)$ laboratory scientists responded (overall response rate 59\%). Free-text comments were received from 19 intensivists, 11 genetic counsellors, 18 clinical geneticists, and four laboratory scientists. Fourteen intensivists and 14 genetic counsellors opted in to follow-up interviews. All were invited, with six and 11, respectively, being interviewed.

Current practice and confidence of intensivists with CMA and ES/GS: All intensivists reported experience ordering CMA, with a third $(28 / 80,35 \%)$ ordering CMA monthly in the past year. In contrast, the vast majority had either never $(39 / 79,49 \%)$ or very rarely (once or twice, $32 /$ $79,40.5 \%$ ) ordered ES/GS. Intensivists rated their confidence higher when using CMA than ES/GS across the range of clinical tasks involved, $p<0.001$ (Fig. 1).

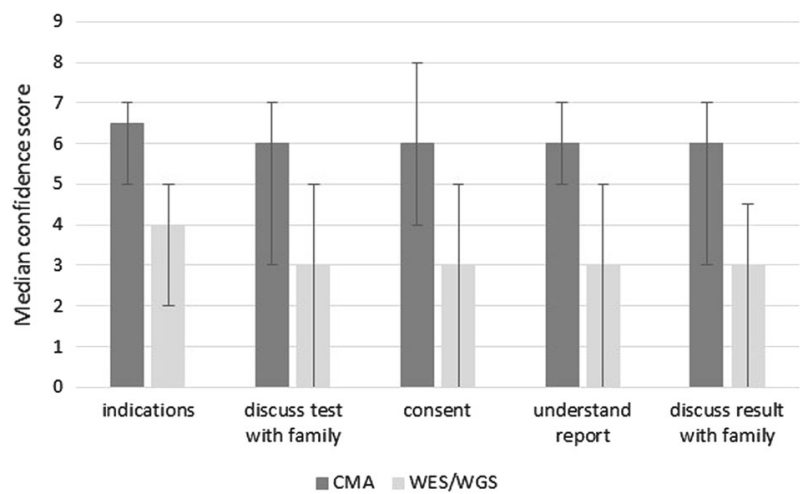

Fig. 1 Intensivist confidence with different aspects of chromosomal microarray (CMA) compared with genomic (ES/GS) testing: understanding test indications, discussing testing with families, consent, understanding reports and discussing results with families. Scale is $0-10$, where 0 is least confident and 10 most confident. Median score and IQR shown

Intensivists commented on their high degree of support for rapid ES/GS:

'I think the day will come when this becomes standard of care' [Intensivist, survey]

'This has huge potential to improve rates of diagnosis of dysmorphic babies or babies with very unusual clinical courses' [Intensivist, survey]

Intensivists explained in interviews that confidence requires exposure through experiential learning.

'My lack of confidence is in the fact that I've been lazy about reading or understanding more, just in terms of competing priorities I think... when we know more about it (ES/GS) we're likely to use more' [Intensivist interview, ID 02] 
Pre-test counseling for genomic tests was further discussed in the interviews with concerns raised including ethical and insurance implications. Australia has a complex, largely publicly funded healthcare system, which guarantees universal access to a range of services; in addition, many Australians choose to have private health insurance. Currently, genetic results do not impact on the ability to obtain private health insurance, but can impact access to other types of insurance such as income protection.

'...Ethics will be an implication... Let us say I find this Huntington's chorea gene carrier, what do I do? This is a two-month-old infant, I've just taken away their life by making that decision to do that test... I feel quite responsible about that... Data confidentiality and privacy is a big deal, and so that is something I, as a clinician, should think about for every patient... I shy away from doing those steps at the moment' [Intensivist interview, ID01]

'(Lower confidence with ES) is more to do with unexpected results... [I] think I've got an idea what the present (status) is regarding insurance and genetic results but doesn't mean it'll always stay that way... My concern there is that I and other clinicians may not have that depth of knowledge for an exome, [Intensivist interview, ID 03]

Perceived clinical utility of rapid genomic tests: When asked in what proportion of NICU/PICU patients tested would rapid results contribute to patient management, a higher proportion of intensivists than clinical geneticists selected 'don't know' (23/79, 29\% v 12/90, 13\%, $p=$ 0.012). For those who provided an estimate, intensivists anticipated a lower clinical utility than clinical geneticists (median $20 \%$ of patients tested vs. $50 \%$, difference in medians $30 \%$, confidence interval around difference $17-43 \%, p<0.001)$. Interviewed intensivists described in further detail their perception of the utility of ES/GS in NICU/PICU (Table 2).

Service delivery model preferences among clinicians: Clinicians were surveyed about their preferred model for delivering rapid genomic testing. All three clinical professional groups expressed a strong preference for a clinical genetics-led service delivery model (Table 3). Qualitative data identified the need for, and value of, a collaborative approach.

'Clinical Geneticists are uniquely placed in knowing when there is likely to be a genetic condition in a patient. Studies have shown we have the highest yield. 
Table 3 Preferences among Australian intensivists, clinical geneticists, and genetic counselors for the model of delivery of a rapid genomic testing service

\begin{tabular}{|c|c|c|c|}
\hline & Intensivists $N=80$ & $\begin{array}{l}\text { Clinical geneticists } \\
N=47\end{array}$ & $\begin{array}{l}\text { Genetic counsellors } \\
N=23\end{array}$ \\
\hline $\begin{array}{l}\text { As inpatient, NICU/PICU team refers to clinical genetics team to initiate } \\
\text { testing and discuss results with families }\end{array}$ & $77.2 \%$ & $87.3 \%$ & $81.8 \%$ \\
\hline $\begin{array}{l}\text { As inpatient, NICU/PICU team initiates testing and discusses results with } \\
\text { families }\end{array}$ & 0 & 0 & 0 \\
\hline \multirow{2}{*}{$\begin{array}{l}\text { As inpatient, NICU/PICU team initiates testing and discusses results with } \\
\text { families, with support from clinical genetics team when needed: }\end{array}$} & $19.0 \%$ & $10.6 \%$ & $18.2 \%$ \\
\hline & Ave. $\pm S D$ & & \\
\hline Advice on whether test is appropriate & $2.9 \pm 1.5$ & $1.8 \pm 1.6$ & $1.8 \pm 1.6$ \\
\hline Consent & $3.7 \pm 1.1$ & $3.2 \pm 1.2$ & $4.3 \pm 1.8$ \\
\hline Interpreting results & $2.1 \pm 1.4$ & $2.0 \pm 0.9$ & $2.0 \pm 0.7$ \\
\hline Discussing results with families & $2.5 \pm 1.3$ & $3.0 \pm 0.9$ & $3.5 \pm 0.9$ \\
\hline Follow-up genetic counseling of family & $1.9 \pm 1.2$ & $2.6 \pm 2.0$ & $3.0 \pm 1.4$ \\
\hline $\begin{array}{l}\text { As outpatient following discharge, clinical genetics team initiates testing } \\
\text { and discusses results with families }\end{array}$ & $1.3 \%$ & $2.1 \%$ & 0 \\
\hline Other & $2.5 \%$ & 0 & 0 \\
\hline
\end{tabular}

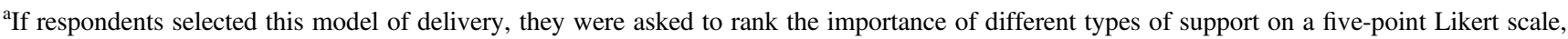
with $1=$ most important, so lower scores indicate higher importance

However this will need to be done in conjunction with the NICU team in a collaborative model. We will have to upskill them in terms of consent, but they are also skilled in discussing results with families' [Clinical geneticist, survey comment]

'Their expertise and their way of working with us and ours with them really enhances patient care' [Intensivist interview, ID03]

However, the boundaries of roles were also evident, with input from a geneticist usually stopping after diagnosis with no further contribution to ongoing management.

'When I invite Genetics to come and review my patient...I know they cannot comment on prognostics ..., which is what the parents want to know... My patient becomes my responsibility.... it will eventually be my team and I who will decide what happens from here on' [Intensivist interview, ID01]

The need for a prompt response from clinical genetics teams was highlighted as critical to the success of a genetics-led model:

'This model is practical if rapid genetics team involvement can be guaranteed. Currently there is often considerable lag between genetic referral and genetic review' [Intensivist, survey]
The second most favored model was intensivist-led, with support from clinical genetics. Both intensivists and genetics professionals identified result interpretation as a key area needing additional support. Intensivists ranked follow-up genetic counseling of family as their top need, whereas clinical geneticists and genetic counselors rated advice on whether the test is appropriate as the area most likely to benefit from their assistance.

'With increasing clinical workload it is more difficult to allocate time to the counseling and therefore this is done by our onsite [genetic] specialists' [Intensivist, survey]

Genetic counselors expressed the need for the model of practice to be adaptive and patient-centered.

'The truth is, some sort of combination (of models) ... would be ideal.... it's such a variable thing, and I don't know that there can be a 'one-size-fits all.' [Genetic counselor interview, ID11]

'It's patient-centered to acknowledge that parents'... needs are going to be multi-faceted, and that not one person can necessarily meet their needs... [It's] very client-centered to think, "Okay, we're going to have a genetic counselor and a clinical geneticist involved for this work, as a team", [Genetic counselor interview, ID8] 


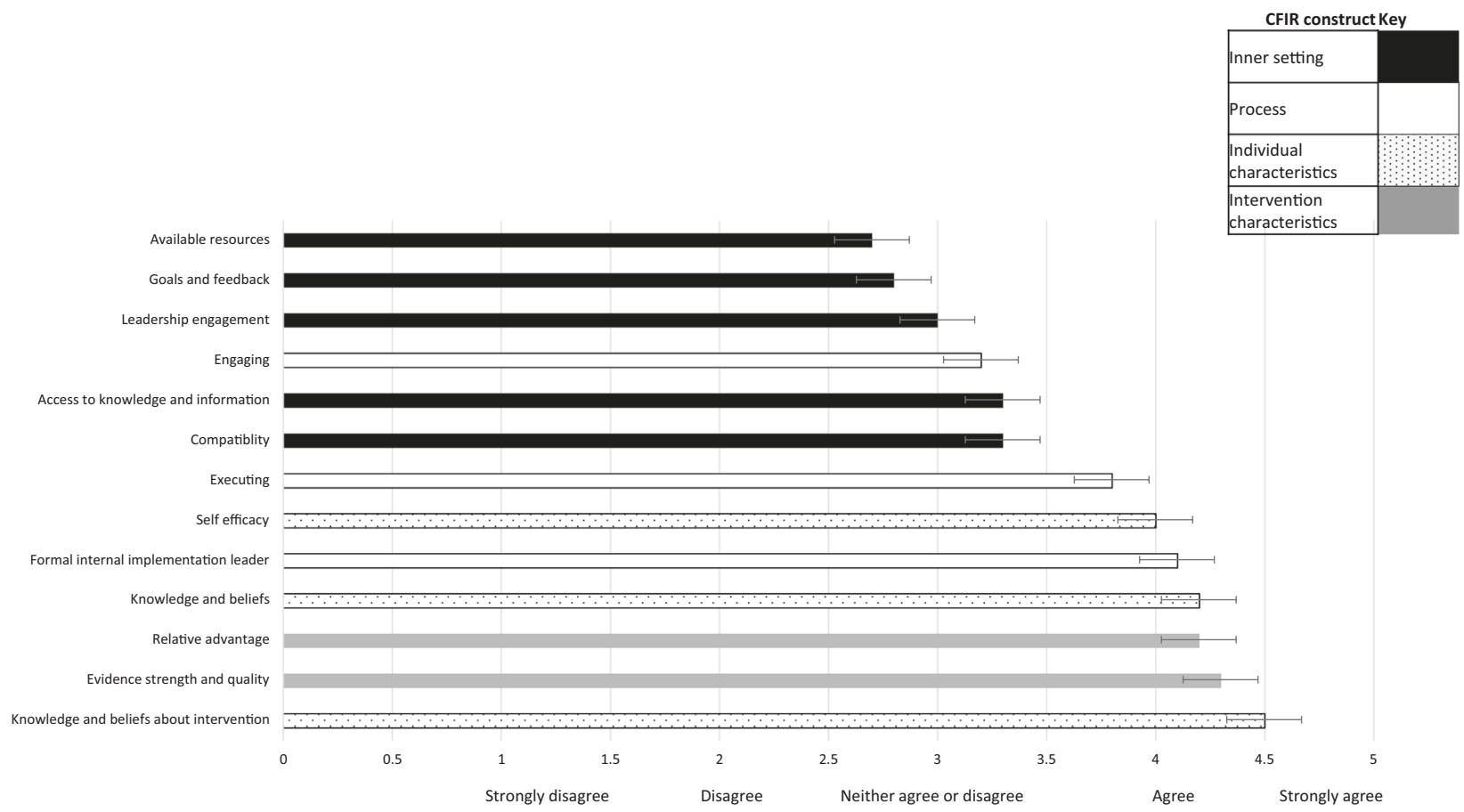

Fig. 2 Clinical geneticist and genetic counselor responses to the IGNITE pre-implementation provider questionnaire based, coded by
Consolidated Framework for Implementation Research constructs. Mean score and standard deviations shown
Perceptions of readiness for implementation: Sixty-eight clinical genetics professionals completed the adapted IGNITE pre-implementation provider questionnaire, which contains 13 statements, and scores ranging from 1 (strongly disagree) to 5 (strongly agree), Supplementary Material. Mean scores ranged from $2.78 / 5$ to $4.5 / 5$ across the 13 statements (Fig. 2). The statements are mapped against constructs from the Consolidated Framework for Implementation Research [19]. The constructs that scored lowest all related to the 'Inner Setting'. This construct includes participants' views on the implementation climate and indicates concern over the organization's readiness for rapid genomic sequencing. Conversely the constructs scoring 4.0/ 5 and above related to the 'Individual Characteristics', 'Intervention Characteristics' and 'Formal Implementation Leader' fields.

Staff from three laboratories completed ORIC $(n=21)$, which has 12 statements: six relating to 'change efficacy' and six to 'change commitment', with scores ranging from 1 (disagree) to 5 (agree), Supplementary Material. Scores demonstrated consistently high 'Change Efficacy' (3.9/5) and high 'Commitment to Change' (4.1/5). These findings suggest that laboratory genetic scientists feel slightly more determined to deliver rapid genomic testing than they feel confident. However, both scores are high, indicating the laboratories surveyed are both ready and able to implement rapid genomic testing. Qualitative concerns were regarding resourcing and organizational readiness.
'I am troubled by the workforce implications of making it sustainable long term. Rapid NGS relies on a wide diversity of skill sets and hence many people in the chain who have to be 'on call' to act immediately should something go wrong. While this can be sustained in a specialized setting with dedicated staff for a short time, rolling it out widely and scaling it up could be very challenging unless the infrastructure and staffing is considered specifically' [Laboratory genetic scientist, survey]

'...We're as equipped as we can be' [Genetic counselor interview, ID10]

'Ultra-rapid' genomic testing in NICU/PICU is changing the way we practice genomics. It is challenging medical professionals (both genetic and non-genetic) to think of genomic sequencing as a firstline test, rather than a back-up plan if all else fails' [Genetic counselor, survey]

'I strongly agree this is important. However, there is the issue of time for the geneticist/counsellor to consent the families and follow up rapid results. We are barely coping with the current number of 
consultations and requests for assistance in the NICU, this would exponentially increase workload on genetics units' [Clinical geneticist, survey]

\section{Discussion}

Genomic testing is an emerging diagnostic modality, which has the potential to overcome many limitations of traditional genetic testing, with particular attractiveness for rare, severe phenotypes in paediatric acute care settings where the contribution of rare Mendelian conditions to disease burden is substantial [20-22]. Demonstrating the ability to deliver genomic testing with rapid turnaround times, coupled with emerging evidence of clinical utility and cost-effectiveness $[3,5,8,9]$, has created momentum for broad roll-out of rapid genomic testing programs in acute paediatric settings internationally. However, concerns have been raised about premature widespread implementation outpacing necessary support, and the potential for this to accelerate ICU clinician moral distress and burnout and damage relationships with families, diminishing the anticipated healthcare benefits [11]. Australian health professionals, who are poised to implement such a program as part of a national network, expressed strong commitment to implementation, counterbalanced by apprehension about organizational readiness and resourcing.

Both intensivists and clinical genetic professionals indicated a strong preference for rapid genomic testing in the acute paediatric setting to be led by clinical genetic services. This may reflect low levels of experience and confidence of intensivists with ES/GS. The low level of workforce preparedness for genomic medicine has repeatedly been identified as a barrier to mainstreaming genomic testing and our findings echo surveys of other subspecialists including oncologists, psychiatrists, neurologists, and cardiologists [23-28]. Sub-specialist surveys have frequently highlighted a strong desire for additional genetics support, especially around result return [29, 30]. Given the potential for genomic test results to be used to make irrevocable decisions about treatment limitation in acute paediatrics, our findings support initial delivery of rapid genomic testing within a genetics-led interdisciplinary team to ensure clinical decisions are based on accurate interpretation of results. However, it would be important that this model is collaborative, and provides opportunities for experiential learning and informal training among the intensive care workforce to ensure timely and appropriate referrals and enable successful transition to another model in the future.

In our study, Australian genetic professionals reported feeling ready and able to implement rapid genomic testing in the NICU/PICU, with implementation constructs relating to 'Individual Characteristics' and 'Intervention Characteristics' scoring highest. These findings suggest genetic professionals think rapid genomic testing will offer a relative advantage over current assessment options in NICU/PICU patients and that they have the necessary knowledge and skills to implement it. Anticipated challenges in integrating rapid genomic testing into practice centered around time and personnel resources, and organizational readiness. The consistent delivery of rapid genomic testing requires multiple changes to established clinical and laboratory work practices, requiring on-demand patient assessment, pre-test counseling, sample processing, data analysis, and result return to all occur within a short timeframe and at unpredictable intervals [5]. Quantifying the additional clinical and laboratory resources required for successful delivery will be important in ensuring appropriate and sustainable funding for rapid diagnosis programs. Furthermore, while many rapid diagnosis programs focus on reporting laboratory turnaround times, data collection on time taken from ICU admission to genomic testing initiation would be an important measure of performance of clinical service delivery models.

Interestingly, Australian intensivists' perception of the clinical utility of genomic testing in ICU was lower than that of clinical geneticists, and lower than the clinical utility reported in the literature $[3,5,8,9]$. This may relate to the previously long turnaround times for genomic testing relative to ICU admission, and/or to overall limited experience of genomic testing among Australian intensivists. The perceived utility of a test influences test adoption [31, 32]. Engagement of the intensive care workforce with genomics will depend on the ability to demonstrate clinical utility in the local setting. To accelerate this, we are creating a learning network where experience is shared between participating sites and professional groups. Balanced presentation of the evidence gathered through the program is necessary to minimize the highs and lows of the hype cycle and promote realistic expectations of both referrers and families.

This is the first study to comprehensively evaluate perspectives and implementation readiness of multidisciplinary teams across multiple sites prior to implementing rapid genomic testing in acute paediatrics. Our findings may not be generalizable to other healthcare systems, particularly those where implementation without on-site clinical genetics services will be required. Capturing the evolution of attitudes and preferences as experience with genomic testing increases will be important in service delivery planning, as will be an assessment of the views of other professionals, in particular intensive care nurses, and other subspecialists, such as neurologists and metabolic physicians.

\section{Conclusion}

Overall, we find strong support for implementation of rapid genomic testing in Australian NICUs/PICUs as part of an 
interdisciplinary service model, which will support a gradual transition of testing to the intensive care workforce. Performance and resource implications of this service delivery model will need to be quantified and addressed to ensure sustainability and efficiency, while addressing gaps in experience, skills, and knowledge among the intensive care workforce will be essential to foster adoption and ensure long-term sustainability of the program.

Acknowledgements This work was supported by the Victorian Government's Operational Infrastructure Support Program and a grant from the Australian National Health \& Medical Research Council (GNT1113531); the contents are solely the responsibility of the individual authors and do not reflect the views of the NHMRC. The authors thank all those who responded to the survey and all members of the Australian Genomics Health Alliance for their contribution to this study.

\section{Compliance with ethical standards}

Conflict of interest The authors declare that they have no conflict of interest.

Ethical approval The Human Research Ethics Committee at The University of Melbourne (HREC 1646785) approved the study.

Publisher's note: Springer Nature remains neutral with regard to jurisdictional claims in published maps and institutional affiliations.

\section{References}

1. Kingsmore SF, Petrikin J, Willig LK, Guest E. Emergency medical genomes: a breakthrough application of precision medicine. Genome Med. 2015;7:82.

2. Daoud H, Luco SM, Li R, Bareker E, Beaulieu C, Jarinova O, et al. Next-generation sequencing for diagnosis of rare diseases in the neonatal intensive care unit. CMAJ. 2016;188:E254-260.

3. Meng L, Pammi M, Saronwala A, Magoulas P, Ghazi AR, Vetrini $\mathrm{F}$, et al. Use of exome sequencing for infants in intensive care units: ascertainment of severe single-gene disorders and effect on medical management. JAMA Pediatr. 2017;171:e173438.

4. Petrikin JE, Cakici JA, Clark MM, Willig LK, Sweeney NM, Farrow EG, et al. The NSIGHT1-randomized controlled trial: rapid whole-genome sequencing for accelerated etiologic diagnosis in critically ill infants. NPJ Genom Med. 2018;3:6.

5. Stark Z, Lunke S, Brett GR, Tan NB, Stapleton R, Kumble S, et al. Meeting the challenges of implementing rapid genomic testing in acute pediatric care. Genet Med. 2018;20:1554-63.

6. Stark Z, Tan TY, Chong B, Brett GR, Yap P, Walsh M, et al. A prospective evaluation of whole-exome sequencing as a first-tier molecular test in infants with suspected monogenic disorders. Genet Med. 2016;18:1090-6.

7. van Diemen CC, Kerstjens-Frederikse WS, Bergman KA, de Koning TJ, Sikkema-Raddatz B, van der Velde JK, et al. Rapid Targeted Genomics in Critically Ill Newborns. Pediatrics. 2017; 140:e20162854.

8. Farnaes L, Hildreth A, Sweeney NM, Clarke MM, Chowdhury S, Nahas S, et al. Rapid whole-genome sequencing decreases infant morbidity and cost of hospitalization. NPJ Genom Med. 2018;3:10.

9. Mestek-Boukhibar L, Clement E, Jones WD, Drury S, Ocaka L, Gagunashvili A, et al. Rapid Paediatric Sequencing (RaPS): comprehensive real-life workflow for rapid diagnosis of critically ill children. J Med Genet. 2018;55:721-8.

10. Willig LK, Petrikin JE, Smith LD, Saunders CJ, Thiffault I, Miller NA, et al. Whole-genome sequencing for identification of Mendelian disorders in critically ill infants: a retrospective analysis of diagnostic and clinical findings. Lancet Resp Med. 2015;3:377-87.

11. Char DS, Lee SS, Magnus D, Cho M. Anticipating uncertainty and irrevocable decisions: provider perspectives on implementing whole-genome sequencing in critically ill children with heart disease. Genet Med. 2018;20:1455-61.

12. Cresswell and Plano Clark. Designing and conducting mixed methods research. SAGE Publications, Thousand Oaks, US, 2018.

13. Sukenik-Halevy R, Ludman MD, Ben-Shachar S, RaasRothschild A. The time-consuming demands of the practice of medical genetics in the era of advanced genomic testing. Genet Med. 2016;18:372-7.

14. National Society of Genetic Counselors. Professional Status Survey, 2016, Vol 2017. http://www.nsgc.org/p/cm/Id/fid=68.

15. Royal College of Physicians. Consultant Physicians Working With Patients. London: RCP, 2013.

16. Nisselle, A, Macciocca I, McKenzie, F, Vuong, H, Dunlop, K, McClaren, B, et al. Readiness of clinical genetic healthcare professionals to provide genomic medicine: an Australian census. J Genet Couns 2018;28:367-377.

17. Shea CM, Jacobs SR, Esserman DA, Bruce K, Weiner BJ. Organizational readiness for implementing change: a psychometric assessment of a new measure. Implement Sci. 2014;9:7.

18. Neuman WL. Social Research Methods; qualitative and quantitative approaches. Pearson Education Inc, Harlow, UK, 2006.

19. Damschroder LJ, Aron DC, Keith RE, Kirsh SR, Alexander JA, Lowery JC. Fostering implementation of health services research findings into practice: a consolidated framework for advancing implementation science. Implement Sci. 2009;4:50.

20. Petrikin JE, Willig LK, Smith LD, Kingsmore SF. Rapid whole genome sequencing and precision neonatology. Semin Perinatol. 2015;39:623-31.

21. Smith LD, Willig LK, Kingsmore SF. Whole-exome sequencing and whole-genome sequencing in critically Ill neonates suspected to have single-gene disorders. Cold Spring Harb Perspect Med. 2015;6:a023168

22. Friedman JM, Bombard Y, Cornel MC, Fernandez CV, Junker $\mathrm{AK}$, Plon SE, et al. Genome-wide sequencing in acutely ill infants: genomic medicine's critical application? Genet Med. 2018. https://doi.org/10.1038/s41436-018-0055-z.

23. Selkirk CG, Weissman SM, Anderson A, Hulick PJ. Physicians' preparedness for integration of genomic and pharmacogenetic testing into practice within a major healthcare system. Genet Test Mol Biomark. 2013;17:219-25.

24. Bonter K, Desjardins C, Currier N, Pun J, Ashbury FD. Personalised medicine in Canada: a survey of adoption and practice in oncology, cardiology and family medicine. BMJ Open. 2011;1: bmjopen-2011-000110.

25. Johansen Taber KA, Dickinson BD. Pharmacogenomic knowledge gaps and educational resource needs among physicians in selected specialties. Pharmgenomics Pers Med. 2014;7:145-62.

26. Gray SW, Hicks-Courant K, Cronin A, Rollins BJ, Weeks JC. Physicians' attitudes about multiplex tumor genomic testing. J Clin Oncol. 2014;32:1317-23.

27. Chow-White P, Ha D, Laskin J. Knowledge, attitudes, and values among physicians working with clinical genomics: a survey of medical oncologists. Hum Resour Health. 2017;15:42-51.

28. Bayefsky MJ, White A, Wakim P, Hull SC, Wasserman D, Chen $\mathrm{S}$, et al. Views of American OB/GYNs on the ethics of prenatal whole-genome sequencing. Prenat Diagn. 2016;36:1250-6. 
29. Johnson L-M, Valdez JM, Quinn EA, Sykes AD, McGee RB, Nuccio $\mathrm{R}$, et al. Integrating next-generation sequencing into pediatric oncology practice: An assessment of physician confidence and understanding of clinical genomics. Cancer. 2017;123:2352-9.

30. Weipert CM, Ryan KA, Everett JN, Yashar BM, Chinnaiyan M, Scott Roberts J, et al. Physician experiences and understanding of genomic sequencing in oncology. J Genet Couns. 2018;27: 187-96.

31. Sanson-Fisher RW. Diffusion of innovation theory for clinical change. Med J Aust. 2004;180:S55-S56.

32. Harris JN, Liljestrand P, Alexander GL, Goddard KA, Kaufmann T, Kolevska T, et al. Oncologists' attitudes toward KRAS testing: a multisite study. Cancer Med. 2013;2:881-8. 